\title{
Surface Based Wireless Power Transmission and Bidirectional Communication for Autonomous Robot Swarms
}

\author{
Travis Deyle \\ Department of Electrical \\ and Computer Engineering \\ Georgia Institute of Technology \\ Atlanta, Georgia 30332 \\ e-mail: tdeyle@gatech.edu
}

\author{
Matt Reynolds \\ Department of Electrical \\ and Computer Engineering \\ Duke University \\ Durham, North Carolina 27708 \\ e-mail: matt.reynolds@duke.edu
}

\begin{abstract}
We introduce an inexpensive, low complexity power surface system capable of simultaneously providing wireless power and bidirectional communication from a surface to multiple mobile robots. This system enables continuous operation of a swarm-sized population of battery-less robots.

Our first prototype consists of a $60 \mathrm{~cm} \times 60 \mathrm{~cm}$ power surface that provides power and bidirectional communication to an initial evaluation group of five test robots, each one consuming $200 \mathrm{~mW}$.

Unlike typical non-resonant inductive (transformer) coupling, power transmission in this system is achieved through magnetic flux coupling between a high $Q$ L-C resonator placed beneath the operating surface and a non-resonant pickup coil on each robot. We explore the design of the pickup coil and conditioning circuitry, and we characterize the position-dependent power density of a static load representative of a small autonomous robot operating on the surface. We demonstrate a continuous power density averaging $4.1 \mathrm{~mW} / \mathrm{cm}^{2}$ for a static load, and develop much greater peak power for dynamic loads via capacitor storage and power conditioning circuitry. We also demonstrate simultaneous broadcast communication between the surface and all robots via amplitude modulation of the magnetic field, and communication between individual robots and the surface via load modulation.
\end{abstract}

\section{INTRODUCTION}

Limited onboard power supplies significantly hamper longterm autonomous mobile robot operation, particularly when working with swarm-sized populations. In these situations, battery life usually varies depending on the activity level of each robot. This adversely affects researchers' ability to test new algorithms and sensor systems both by creating troublesome logistical barriers and preventing the evaluation of large numbers of robots or algorithms with long running times.

With swarm robotics, as in mobile robotics in general, robot power budget strongly influences mechanical and electrical design, operational duration, and even behavior. We have been developing methods to enable continuous robot operation by eliminating recharging, battery swapping, and cumbersome tethers altogether. Previous solutions in the literature have relied on tethers, battery exchange, docking or recharging

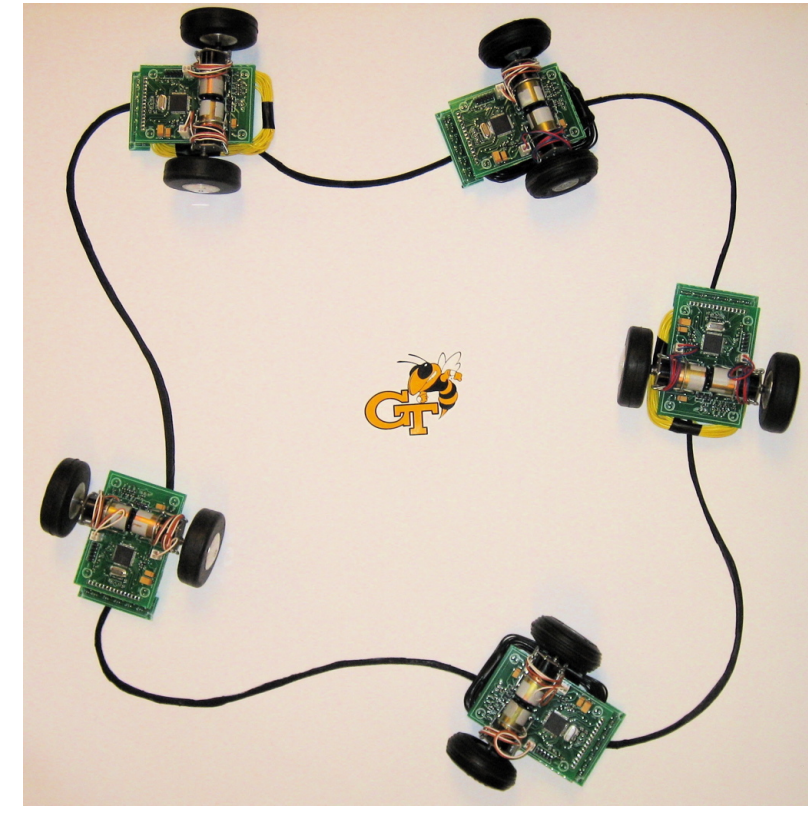

Fig. 1. Swarm Operating Battery-Free on the Power Surface

schemes, or specialty power sources such as fuel cells or hydrocarbon engines. In contrast to these approaches, we propose a low-complexity, inexpensive wireless power system capable of simultaneously providing wireless power and bidirectional communication to a swarm of mobile robots in continuous operation on a bounded surface. We have built and tested a demonstration system consisting of five mobile robots, each drawing $200 \mathrm{~mW}$ of power from a $60 \mathrm{~cm} \times 60 \mathrm{~cm}$ power surface.

We characterize the position dependence of power density on the surface, and demonstrate an average received power density of $4.1 \mathrm{~mW} / \mathrm{cm}^{2}$. In comparison, bright sunlight has a power density of approximately $20 \mathrm{~mW} / \mathrm{cm}^{2}$ after typical $20 \%$ solar cell conversion efficiency, while indoor light levels are typically 1-2 orders of magnitude less.

We also demonstrate simultaneous multicast communication 


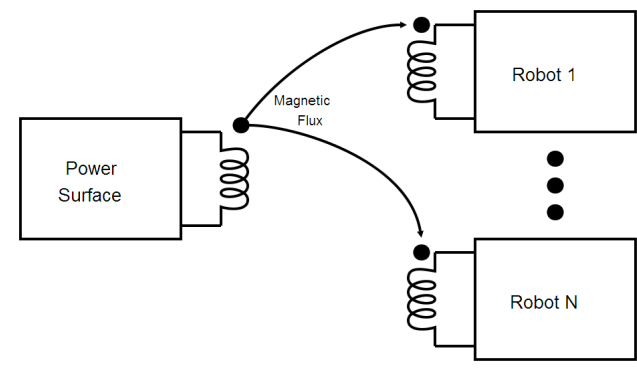

Fig. 2. Conceptual Power Surface System Diagram

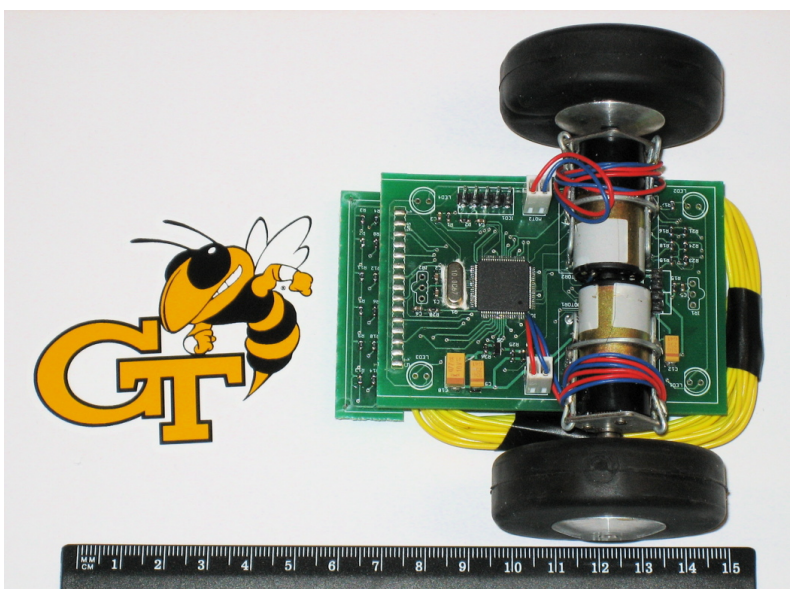

Fig. 3. Close-up view of test robot

to all robots on the surface using amplitude modulation of the $112 \mathrm{kHz}$ carrier. Individual robots communicate back to the surface drive circuitry via a load-modulation technique. We estimate the cost of the power reception and conditioning circuitry on each mobile robot at approximately $\$ 3.00$ per robot in 100 robot quantities, and we expect this to compare very favorably with the cost of lithium ion batteries and their associated charge control circuitry.

\section{RELATED WORK}

Many techniques have been developed to overcome the problems of limited battery capacity in autonomous mobile robots. One method is autonomous docking with a recharging station [1] that makes direct electrical contact with the robot. This method is commonly used in commercial robots such as iRobot's Roomba vacuuming robot. Because of the impracticality of simultaneously docking large numbers of robots, as would be required to dock and recharge an entire swarm at once, various battery exchange behaviors such as those described in [2] have also been explored. Unfortunately, these techniques still suffer from the inherent drawbacks of onboard battery power, most notably the requirement that robot behavior be modified to include recharging or battery exchange, and an inability to support continuous operation of the entire group of robots.
Continuous inductive wireless power transfer and communication has long been employed in radio frequency identification (RFID) tags [3], [4], as well as subcutaneous medical implants [5], [6]. For these applications, significant effort has been invested to optimize transmitting and receiving coil geometry, power conditioning circuitry, and communication circuitry and protocols. Most of the RFID and medical implant communication and charging systems are designed for relatively low power densities, and in the case of medical implants, the relative position of the transmitting and receiving coils are fixed by design.

A few wireless power systems have recently been designed for robotic applications. For example, one recent system relies on a large array of smaller coils driven by MEMS switches and organic field-effect transistors to selectively transmit wireless power with relatively high efficiency by selectively enabling only those transmitting coils in the immediate vicinity of the powered device [7]. An alternative system, developed for the MiCRoN Project [8] relies on a series of multi-phased coils to transmit power to a number of micro-robots. These systems are well designed for the purpose of supplying power to very small robots, but they both require relatively complex control schemes to prevent destructive interference in the electromagnetic field, and provide limited power densities due to their small coils. Additionally, the complex multiple-coil geometry results in a relatively high fabrication cost when compared with the single-resonator system presented in this work.

Finally, there is a new class of micro-robots that utilize wireless power coupling in a "logic-less" fashion by using a spatially addressible electrostatic or magnetostatic field to drive MEMS-sized electrostatic or magnetostrictive actuators [9], [10]. While these robots are entirely wirelessly powered, they do not include on-board sensing and computation, thus rendering them unsuitable for research in distributed sensing and control algorithms.

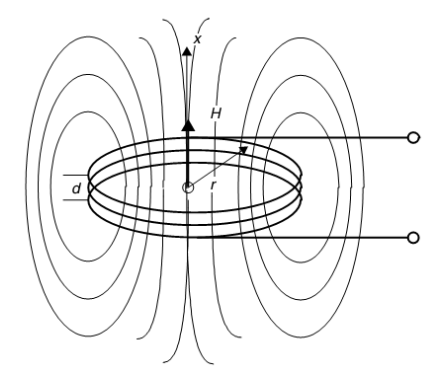

Fig. 4. Magnetic field created by a round coil, from [3]

\section{Operating PRINCIPLES}

There are numerous sources that describe the underlying physics of inductive power transfer, including efficiency and design considerations, e.g. [3], [4], [5]. At the heart of the system is a resonant transmitting coil, which by Ampere's law generates a magnetic field due to the current flowing through 
each turn of wire. The current $I$ flowing through a round coil of radius $r$ results in a magnetic field strength $H$ at a certain distance $x$ from the center of the coil given by

$$
H(x)=\frac{I \cdot N_{t} \cdot r^{2}}{2 \sqrt{\left(r^{2}+x^{2}\right)^{3}}}
$$

where $I$ is the circulating current in the transmitting coil, and $N_{t}$ is the number of turns in the transmitting coil. The geometry used in this expression is shown in Figure 4.

Because the transmitting coil is resonant, the circulating current in the transmitting coil is larger by $Q$ than the drive current supplied to the coil. In our $60 \mathrm{~cm} \times 60 \mathrm{~cm}$ power surface, the transmitting coil has 23 turns, a measured inductance of $740 \mu \mathrm{H}$, and a measured DC resistance of $0.42 \Omega$. At the operating frequency of $112 \mathrm{KHz}$, the calculated coil $Q=2 \pi f L / R=1240$. Losses in the mica resonating capacitors as well as in proximate materials limit the loaded $Q$ of the resonant circuit to a measured value of $Q \approx 117$.

When the transmitting coil is much larger than the pickup coil, as in our power surface where the transmitting coil has an area of $3600 \mathrm{~cm}^{2}$ while the pickup coil has an area of only $78.5 \mathrm{~cm}^{2}$, we can assume to a first order that $H$ is uniform within the pickup coil, although $H$ is definitely not uniform as the pickup coil is moved about on the power surface. The magnetic field $H$ induces an electric potential $E$ across the non-resonant pickup coil given by Faraday's law:

$$
E=\mu_{0} H \omega N_{p} A_{p}
$$

where $\mu_{0}=4 \pi \cdot 10^{-7} \mathrm{H} / \mathrm{m}, N_{p}$ is the number of turns in the pickup coil, $\omega$ is the operating frequency, and $A_{p}$ is the area of the pickup coil.

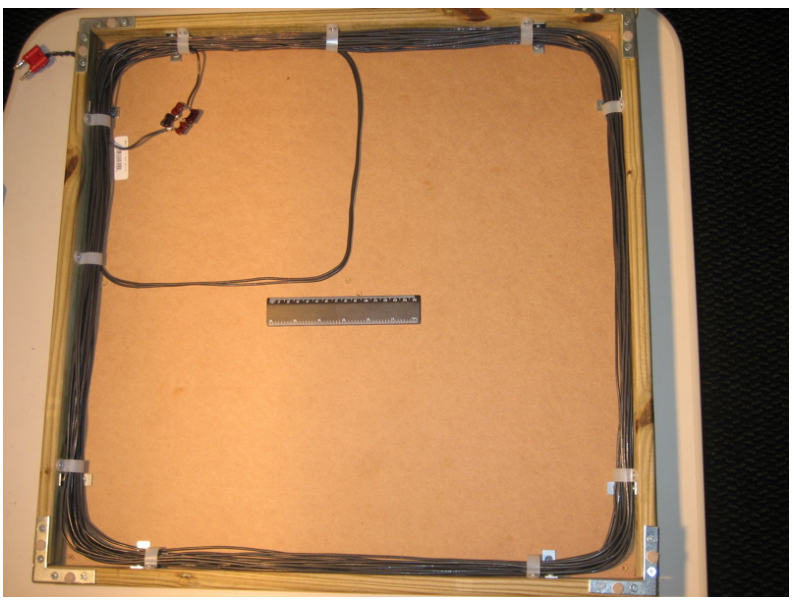

Fig. 5. Power surface resonator and primary coil

\section{System Architecture And Design}

In our system, power transmission is achieved by exciting a large, high $Q$ L-C resonator located just beneath the top of the power surface as shown in Figure 5. This results in a smooth operating surface, in contrast to the typical nonresonant transformer coupling used for most wireless chargers, including the commonly used inductive toothbrush chargers. In typical non-resonant transformer coupled applications, the transmitting coil protrudes from the charger and is inserted into the receiving coil to maximize mutual coupling. This is undesirable in mobile robot applications as the robots need to receive power regardless of their location on the power surface.

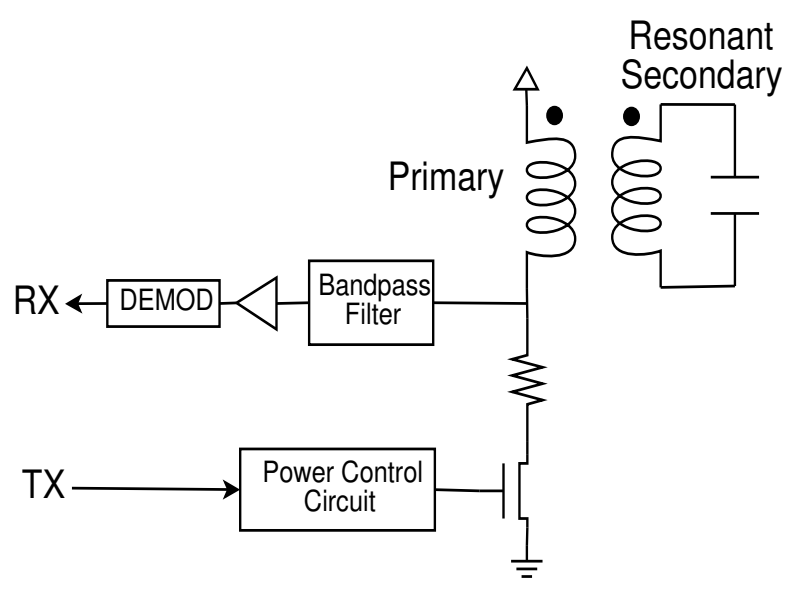

Fig. 6. Power surface transmitter and receiver circuitry

\section{A. Power Surface Design}

As shown in the power surface transmitter and receiver schematic, Figure 6, the transmitting resonator is excited by a 2-turn primary coil in order to minimize the effect of the drive circuit on resonator $Q$. An n-channel power MOSFET with a low on-state resistance of $58 \mathrm{~m} \Omega$ pulls current from a $12 \mathrm{~V}$ DC source through the primary coil on every half cycle of the $112 \mathrm{KHz}$ transmission frequency. The resonator 'rings' on negative going half cycles of the transmission frequency when the FET switch does not conduct. The prototype power surface consumes a quiescent power of $12 \mathrm{~W}$ to overcome losses in the finite $Q$ of the resonant circuit. The resulting magnetic flux is coupled to a non-resonant pickup coil located on each robot. Because robots carry non-resonant pickup coils, they convert the magnetic field passing through their pickup coils to an induced voltage without influencing the resonant frequency of the excitation coil or other robots that may be nearby.

The transmitter's operating frequency is produced by a PWM output from a Microchip Inc. PIC18F452 microcontroller operating at $40 \mathrm{MHz}$. The microcontroller's timer unit is used to divide the $40 \mathrm{MHz}$ clock down to the achievable PWM frequency closest to the desired $112 \mathrm{KHz}$ resonant frequency. The microcontroller can also be used to transmit data to the robots on the surface by amplitude modulating the surface's AC magnetic field. This is accomplished by varying the PWM duty cycle which in turn varies the on-time of the switching FET and therefore modulates the current flowing in the primary of the transmitting resonator.

Data may be received from the robots by means of load modulation. A load modulation switch on each robot varies 
the amount of current drawn from the surface. An envelope detector in the power surface controller detects the load modulation, which is filtered by an op-amp based active filter, amplified, and thresholded to present a data signal to the surface controller's microcontroller.

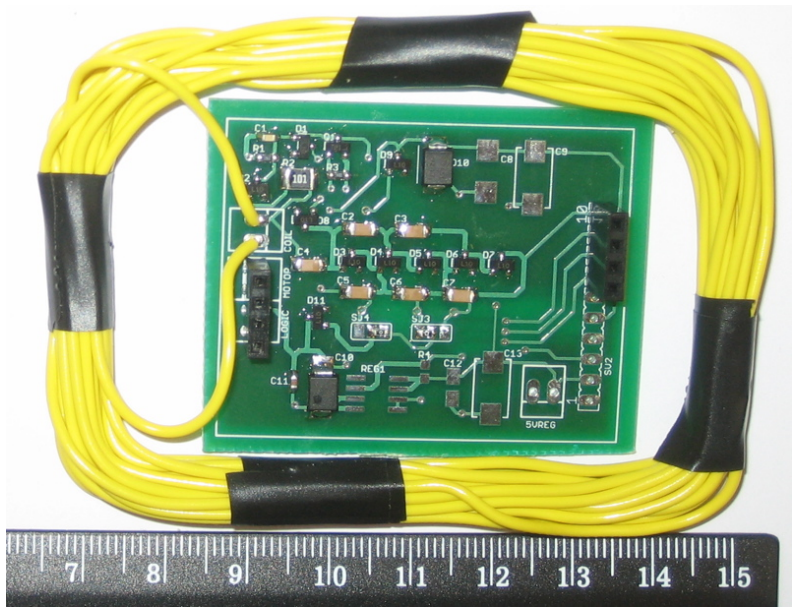

Fig. 7. Individual robot power circuit board

\section{B. Robot power and bidirectional communication circuitry}

The robot pickup coil and conditioning circuitry, shown in Figure 7 , is designed to provide maximum power transfer at a desired voltage and load current, while simultaneously preventing multiple-load interference, which is important with robots where logic and motors must operate simultaneously. This circuit, shown schematically in Figure 8, consists of three main parts.

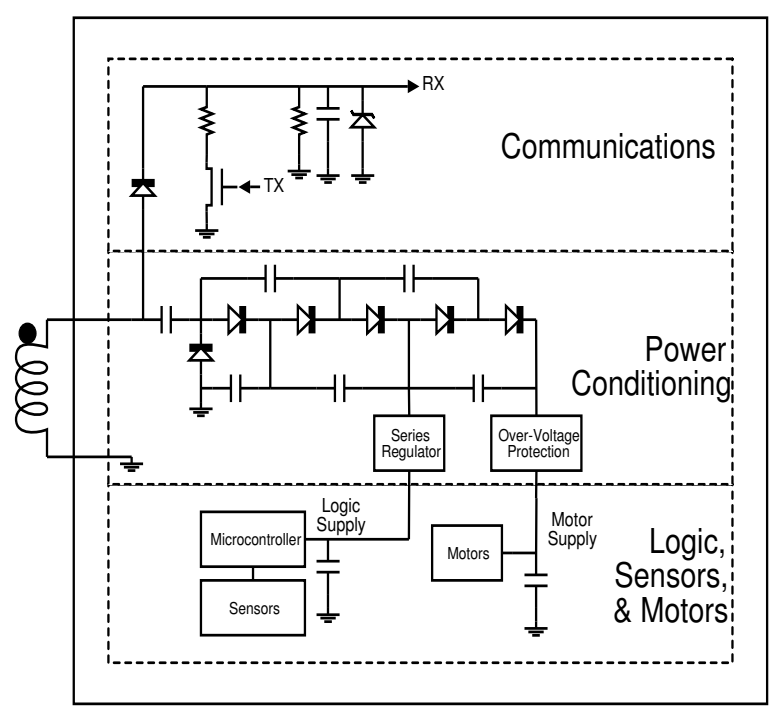

Fig. 8. Individual robot power receiving and bidirectional communication circuitry
1) Power Rectification and Regulation: The power rectification and conditioning circuit consists of a cascaded voltage multiplier using low- $V_{f}$ Schottky diodes to multiply and rectify the AC voltage provided by the receiving coil. Two distinct voltages are generated, one at $4 \cdot V_{\text {coil }}$ to be regulated down to $5 \mathrm{~V}$ for the microprocessor and logic supply, and another at $6 \cdot V_{\text {coil }}$ to be used directly by the motor driver without further regulation beyond a $9 \mathrm{~V}$ Zener clamp to provide overvoltage protection.

Separating the logic and motor power by means of a chain of voltage multipliers, and separately regulating logic power, ensures that logic power is maintained even under varying motor power loads. Because each subsequent stage of the voltage multiplier can only draw power when the prior stage voltage exceeds its diode turn-on voltage, excessive motor current drain does not affect the availability of logic power. This arrangement also allows the motors to be operated at a voltage yielding peak motor efficiency.

2) Communications circuitry: To enable bidirectional communication with the power surface, simple transmitting and receiving circuits are included. An envelope detector with a fast time constant $(\tau \approx 33 \mu \mathrm{s})$ detects rapid changes in the power surface's transmitted field, which are filtered and thresholded by a comparator in the robot's microcontroller. A load modulating FET switches a $100 \Omega$ load resistor across the pickup coil to send data from the robot to the surface's microcontroller.

\section{Power transfer eVAluation}

We have characterized the available power from the power surface with a number of different pickup coil configurations. We ultimately constructed a coil of 15 turns, with $5 \mathrm{~cm}$ radius $\left(\approx 78.5 \mathrm{~cm}^{2}\right)$ and made from 24 AWG wire. This combination is optimized for the test robots' power requirement of approximately $300 \mathrm{~mW}$ peak. Power consumption of our test robots is dominated by two efficient miniature gearhead motors, each drawing about $30 \mathrm{~mA}$ at $5 \mathrm{~V}$, or approximately $300 \mathrm{~mW}$ total. For the purpose of power surface characterization, we substituted an $80 \Omega$ resistive load, which closely approximates the motor's load characteristics.

Available power was measured for varying XY positions (Figure 9) and heights (Figure 10) above the power surface. As expected from Equation 1, the highest power density is obtained at $x \approx 0$, directly atop the power surface. Within the active region of the power surface (the area enclosed by the transmitting resonator), the minimum power at any location was $150 \mathrm{~mW}$, the maximum was $529 \mathrm{~mW}$, and the average power was $318 \mathrm{~mW}$. This equates to a power density of $\approx 4.1 \mathrm{~mW} / \mathrm{cm}^{2}$ over the $78.5 \mathrm{~cm}^{2}$ coil.

In order for a swarm to operate continuously and simultaneously, it is important that there be little or no interaction between individual power-receiving coils. The lack of coil interaction was demonstrated by moving pairs of coils as shown in Figure 11. As shown in Figure 12, there is little change in available power until the two coils physically overlap. 

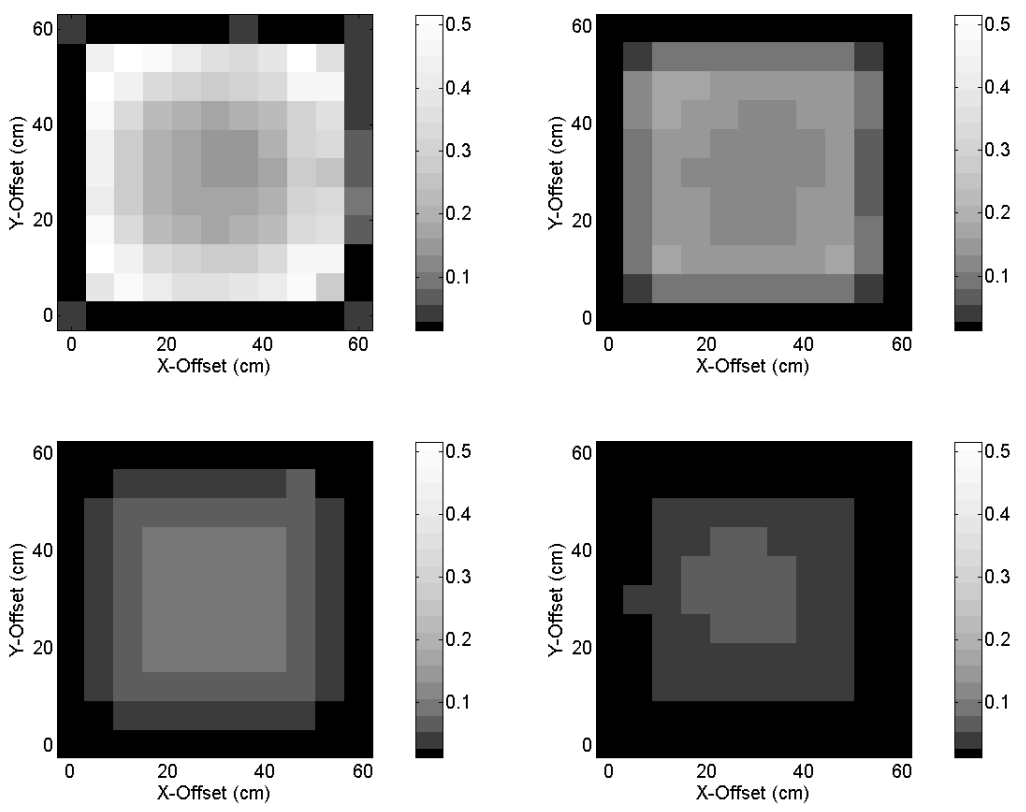

Fig. 9. Power into $80 \Omega$ load, $N_{p}=15, A_{p}=78.5 \mathrm{~cm}^{2}$. Top Left: $x=0 \mathrm{~cm}$ (on surface) Top Right: $x=5 \mathrm{~cm}$ Bottom Left: $x=10 \mathrm{~cm}$ Bottom Right: $x=15 \mathrm{~cm}$

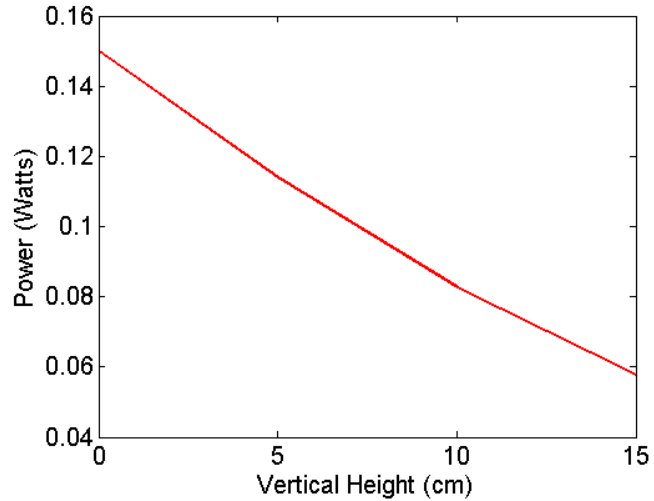

Fig. 10. Available power versus height $x, N_{p}=15, A_{p}=78.5 \mathrm{~cm}^{2}$

\section{A. Communication}

We have also evaluated bidirectional communication. Communicating from the robot to the surface via load modulation is shown in Figure 13, where load modulation induces a small but detectable amplitude modulation depth of $\approx 2 \%$ of the drive coil's voltage; this is sufficient to ensure recovery by the data demodulator. Surface-to-receive coil communication was evaluated by amplitude modulating the drive coil's power and demodulating this signal as shown in Figure 14. Though we have not explicitly examined communication and arbitration/collision protocols, a variety of solutions have been explored in the RFID literature [3], [4].

\section{B. Robot Swarm Test}

We constructed a mini-swarm of five battery-free, wirelessly powered autonomous mobile robots, as shown in Figure 1. A close-up view of an individual robot is shown in Figure

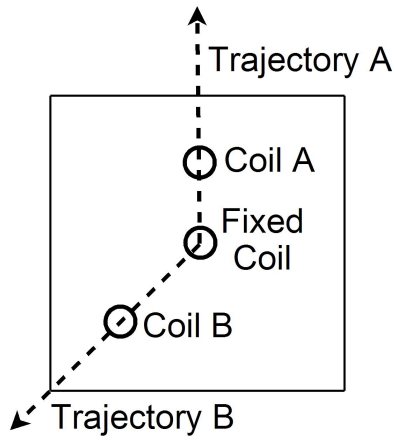

Fig. 11. Path of pickup coil interaction experiments

3. Each robot consists of a PIC18F8527 microcontroller, 6 Omron EE-SY125 infrared reflective sensors, and the power supply circuitry shown in Figure 7. Each robot also possesses higher bitrate infrared peer-to-peer communication capabilities independent of the surface. To demonstrate the utility of the wireless power system for autonomous swarms, each robot executes a simple line following behavior using linear proportional control. With this group of robots we have demonstrated unlimited run time.

\section{CONCLUSION}

We introduce an inexpensive, low complexity power surface system capable of simultaneously providing wireless power and bidirectional communication from a surface to multiple mobile robots. This system enables continuous operation of a swarm-sized population of battery-less robots.

Our first prototype consists of a $60 \mathrm{~cm} \times 60 \mathrm{~cm}$ power surface that provides power and bidirectional communica- 

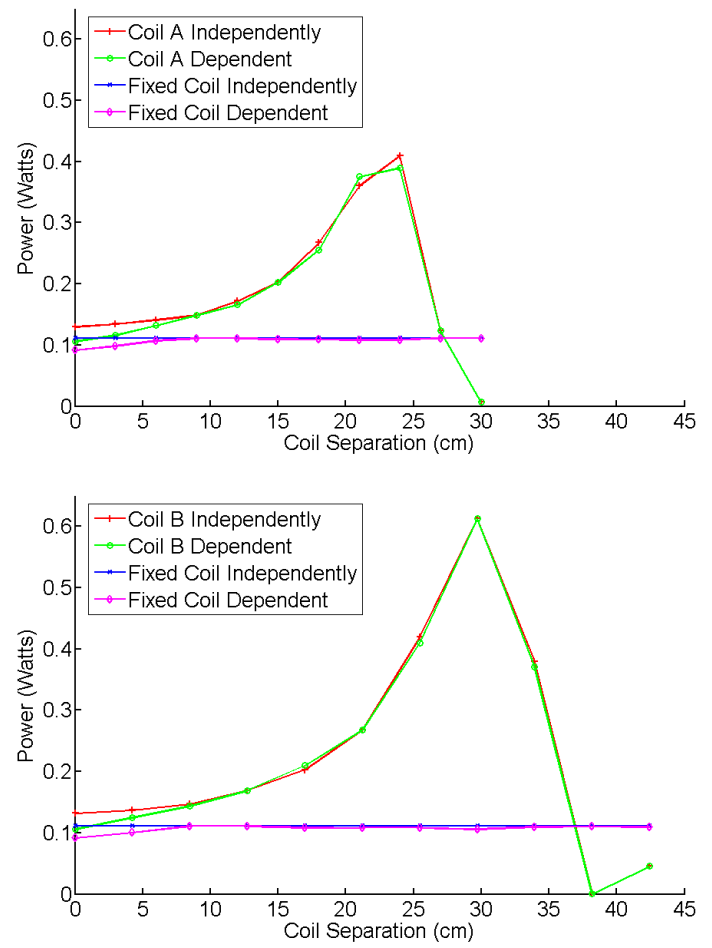

Fig. 12. Interaction between two coils, $N_{p}=15, A_{p}=78.5 \mathrm{~cm}^{2}$, load $80 \Omega$. Top: Fixed coil power with trajectory A Bottom: Fixed coil power with trajectory B

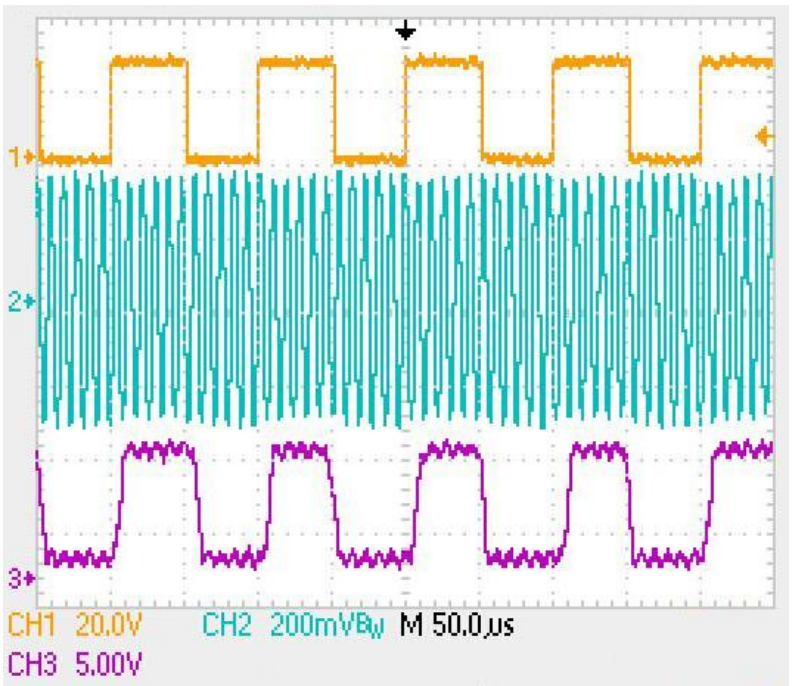

Fig. 13. Robot-to-surface communication at a bitrate of 20kbps. Top: Robot's transmitted data Middle: Input to power surface demodulator Bottom: Output of power surface demodulator

tion to an initial evaluation group of five test robots, each one consuming $200 \mathrm{~mW}$. We demonstrate a continuous power density averaging $4.1 \mathrm{~mW} / \mathrm{cm}^{2}$ for a static load, and develop much greater peak power for dynamic loads via capacitor storage and power conditioning circuitry. We also demonstrate simultaneous broadcast communication between the surface and all robots via amplitude modulation of the magnetic field,

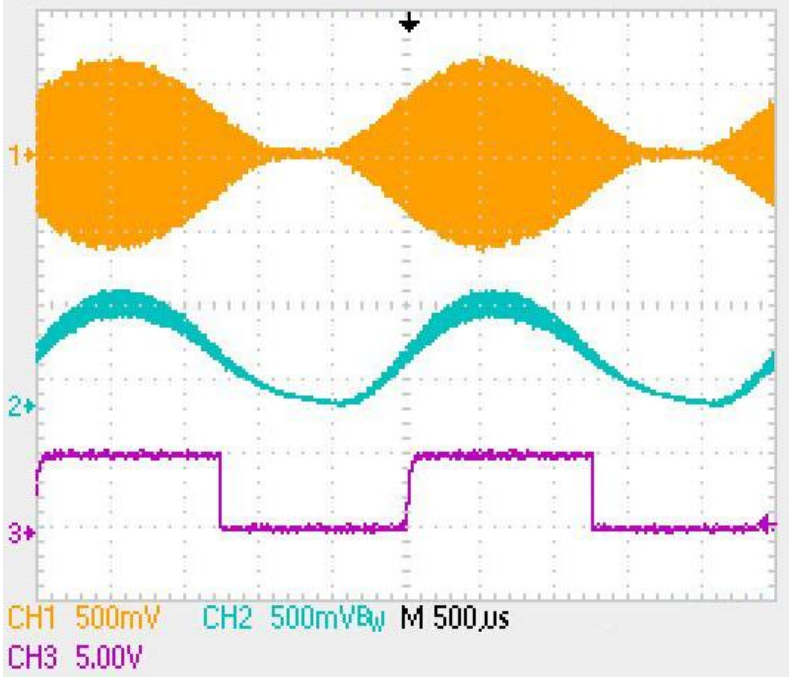

Fig. 14. Surface-to-robot communication at a bitrate of 800bps. Top: Surface field modulation Middle: Robot's demodulator outputBottom: Robot's thresholded data

and communication between individual robots and the surface via load modulation.

\section{REFERENCES}

[1] M. Silverman, D. Nies, B. Jung, and G. Sukhatme, "Staying alive: a docking station for autonomous robot recharging," IEEE International Conference on Robotics and Automation (ICRA), vol. 1, pp. 1050-1055, 2002.

[2] T. D. Ngo, H. Raposo, and H. Schioler, "Potentially distributable energy: Towards energy autonomy in large population of mobile robots," International Symposium on Computational Intelligence in Robotics and Automation (CIRA), pp. 206-211, 2007.

[3] K. Finkenzeller, RFID Handbook: Fundamentals and Applications in Contactless Smart Cards and Identification. Wiley, 2003.

[4] B. Jiang, J. R. Smith, M. Philipose, S. Roy, K. Sundara-Rajan, and A. V. Mamishev, "Energy scavenging for inductively coupled passive rfid systems," IEEE Transactions on Instrumentation and Measurement, vol. 56, no. 1, pp. 118-125, 2007.

[5] G. Wang, W. Liu, M. Sivaprakasam, M. Humayun, and J. Weiland, "Power supply topologies for biphasic stimulation in inductively powered implants," IEEE International Symposium on Circuits and Systems (ISCAS), vol. 3, no. 12, pp. 2743-2746, 2005.

[6] P. Li, J. Principe, and R. Bashirullah, "A wireless power interface for rechargeable battery operated neural recording implants," 28th Annual International Conference of the IEEE Engineering in Medicine and Biology Society (EMBS), pp. 6253-6256, 2006.

[7] T. Sekitani, M. Takamiya, Y. Noguchi, S. Nakano, Y. Kato, K. Hizu, H. Kawaguchi, T. Sakurai, and T. Someya, "A large-area flexible wireless power transmission sheet using printed plastic mems switches and organic field-effect transistors," International Electron Devices Meeting (IEDM), pp. 1-4, 2006.

[8] J. Gao, "Inductive power transmission for untethered micro-robots," 31st Annual Conference of IEEE Industrial Electronics Society (IECON), pp. 6-11, 2005.

[9] P. Basset, L. Buchaillot, A. Kaiser, and D. Collard, "Design of an autonomous micro robot," 8th IEEE International Conference on Emerging Technologies and Factory Automation, vol. 2, pp. 795-798, 2001.

[10] T. Fukuda, H. Hosokai, H. Ohyama, H. Hashimoto, and F. Arai, "Giant magnetostrictive alloy (gma) applications to micro mobile robot as a micro actuator without power supply cables," Proceedings Micro Electro Mechanical Systems (MEMS), pp. 210-215, 1991. 